\title{
Taylor expansion of luminosity distance in Szekeres cosmological models: Effects of local structures evolution on cosmographic parameters
}

\author{
Mattia Villani \\ Sezione INFN di Firenze, \\ Polo Scientifico Via Sansone 1, 50019, Sesto Fiorentino (FI), Italy \\ E-mail: villani@fi.infn.it
}

\begin{abstract}
We consider the Goode-Wainwright representation of the Szekeres cosmological models and calculate the Taylor expansion of the luminosity distance in order to study the effects of the inhomogeneities on cosmographic parameters.

Without making a particular choice for the arbitrary functions defining the metric, we Taylor expand up to the second order in redshift for Family I and up to the third order for Family II Szekeres metrics under the hypotesis, based on observation, that local structure formation is over.

In a conservative fashion, we also allow for the existence of a non null cosmological constant.
\end{abstract}

Keywords: Szekeres metrics, luminosity distance, dark energy 


\section{Contents}

1 Introduction $\quad 1$

2 Szekeres cosmological models $\quad 2$

2.1 Family I $\left(\beta,,_{r} \neq 0\right) \quad 3$

2.2 Family II $\left(\beta,{ }_{r}=0\right) \quad 4$

2.3 Density contrast and shear 4

2.4 Cosmographic parameters 5

2.5 Null geodesics and redshift 5

3 Luminosity distance $\quad 6$

$\begin{array}{lll}3.1 & \text { Family I Szekeres cosmological models } & 7\end{array}$

3.2 Family II Szekeres cosmological models 8

4 Angular dependence $\quad 9$

4.1 The effective deceleration parameters $\quad 9$

$\begin{array}{lll}4.2 & \text { The effective jerk parameter } j_{\text {eff }} & 10\end{array}$

5 Comparison with observations $\quad 11$

5.1 Effects of angular dependency 12

6 Conclusions 12

$\begin{array}{ll}\text { A Useful relations } & 13\end{array}$

$\begin{array}{lr}\text { B Christoffel symbols } & 14\end{array}$

$\begin{array}{lr}\text { C Null geodesics and redshift } & 16\end{array}$

\section{Introduction}

At the end of the nineties, it was proven that the Universe expansion is accelerating $[1,2]$.

Assuming that General Relativity (GR) is the theory of gravitation (valid at every scale, from the Solar System to galaxy clusters), in order to explain this phenomenon one needs a new form of energy with negative pressure able to contrast gravitational attraction which, otherwise, would slow down expansion.

The most successful model proposed in order to explain observations is $\Lambda$ CDM: the Universe is assumed (at large scales) homogeneous and isotropic; it is described by a flat Friedmann-Lemaitre-Robertson-Walker (FLRW) metric; along with baryonic matter it contains a component of cold dark matter (in order to explain spiral galaxies rotation curves and structures formation) and a component of dark energy described by the cosmological constant $\Lambda$. At present time, the dark energy amounts to about $68 \%$ of the content of the Universe [5].

Even if $\Lambda \mathrm{CDM}$ is in very good agreement with observations (see [5] for recent results), it has some flaws (see [7, 59], for example): the cosmological constant is usually interpreted in terms of vacuum energy, but its value is way too small: it is known, in fact, that there is a 
difference of over 100 orders of magnitude between the theoretical prediction and the actual value of $\Lambda$; furthermore the acceleration starts at redshifts close to zero (the coincidence problem) and it is very difficult to explain why in $\Lambda$ CDM model.

In the literature, numerous different approaches have been proposed in order to explain the acceleration and overcome $\Lambda \mathrm{CDM}$ problems: some try to devise a new theory of gravity, like $f(R)$ theories (see for example [6]); other consider new forms of energy or fields that have the same effects as the cosmological constant.

Another approach is to consider the effects of inhomogeneities: it is evident that, at least at small scales, the universe is not homogeneous and isotropic, therefore it is natural to ask whether inhomogeneities can have some effect on the expansion. Among these approaches we mention the cosmological backreaction (see for example [11] - [20] for an incomplete list), and the use of non homogeneous metrics solution of the Einstein's equations (see [22]-[42] among the others and [49] and [50] for reviews on the argument).

In the former, one studies the effects of local structure formation and evolution on the cosmic expansion. In the latter, one studies the effect of inhomogeneities on observations.

A good review of all these approaches is [7].

In this paper we follow the last approach and use Szekers' models, exact non homogeneous solution of the Einstein's equations with non rotational dust. In a conservative fashion, we consider a non null cosmological constant.

In particular we work with the Goode-Wainwright representation, in which it is evident that Szekeres models describe non-linear perturbation of a FLWR background.

Our aim is to calculate the Taylor expansion of luminosity distance of Szekeres models in order to study the effects of inhomogeneities and structure evolution on the deceleration and jerk parameters.

This paper is organized as follows: in section 2, we briefly review the properties and the definition of Szekeres models and define the redshift; in section 3 we calculate the Taylor expansion of the luminosity distance; in section 4 we define our effective cosmographic parameters ans discuss their direction dependence; in section 5 we compare our effective parameters with the observed ones and discuss the effects that local inhomogeneities and local structures evolution have on the acceleration; in the appendix A, we report some relations needed in our calculation; in the appendix B, we report the Christoffel symbols of the considered metrics; and in appendix $\mathrm{C}$ we calculate null geodesics.

We assume $8 \pi G=c=1$.

\section{Szekeres cosmological models}

Szekeres cosmological models are solutions of the Einstein's Field equations of the type: ${ }^{1}$

$$
d s^{2}=d t^{2}-e^{-2 \alpha} d r^{2}-e^{-2 \beta}\left(d x^{2}+d y^{2}\right)
$$

with irrotational dust as a source (see [43] - [50]). One can also include a cosmological constant as a source (see for example [49] and [52]) ${ }^{2}$. In their most general form they have no killing vectors [51] and are therefore inhomogeneous. ${ }^{3}$

\footnotetext{
${ }^{1}$ Coordinates in (2.1) are stereographic projection ([48]-[50]).

${ }^{2}$ Szafron [44] (see also [48-50]) considered radiation too, but we won't consider that.

${ }^{3}$ Particular choices for the arbitrary functions can, nevertheless, lead to models with symmetries: all Friedmann-Robertson-Walker and Lemaître-Tollman-Bondi models can be obtained from the Szekeres ones (see [49] on this topic).
} 
There are two families of this metric: the one with $\beta,_{r} \neq 0$ (Family I) and the one with $\beta, r=0$ (Family II). The function $\alpha$ always depends on all of the variables.

Goode and Wainwright gave a representation of the metric for the case $\Lambda=0$ in which it is evident that the models describe non linear perturbations of the FLRW metrics. It also encompasses properties of both families [47]. The metric is given by:

$$
d s^{2}=d t^{2}-S^{2}\left[H^{2} W^{2} d r^{2}+e^{2 \nu}\left(d x^{2}+d y^{2}\right)\right] .
$$

The function $S(t, r)$ is the solution of the equation:

$$
\left(\frac{\dot{S}}{S}\right)^{2}=\frac{2 \mathcal{M}}{S^{3}}-\frac{\kappa}{S^{2}} \quad \kappa=\{0, \pm 1\},
$$

where $\mathcal{M}(r)$ is an arbitrary function linked to the matter density (see later); in case a cosmological constant is present, we have [52]:

$$
\left(\frac{\dot{S}}{S}\right)^{2}=\frac{2 \mathcal{M}}{S^{3}}+\frac{\Lambda}{3}-\frac{\kappa}{S^{2}}, \quad \kappa=\{0, \pm 1\} .
$$

With or without a cosmological constant, we have:

$$
\begin{aligned}
H(t, x, y, r) & =A(t, x, y, r)-F(t, r)= \\
& =A(t, x, y, r)-\left[\beta_{+}(r) f_{+}(t)+\beta_{-}(r) f_{-}(t)\right],
\end{aligned}
$$

where $f_{ \pm}(t)$ are independent solutions of the (Raychaudhuri) equation: ${ }^{4}$

$$
\ddot{F}+2 \frac{\dot{S}}{S} \dot{F}-\frac{3 \mathcal{M}}{S^{3}} F=0
$$

and the $\beta_{ \pm}(r)$ functions are arbitrary: the choice $\beta_{ \pm} \equiv 0$ leads to Robertson-Walker models, written in an unusual coordinate system (see [49]).

The two families are defined as follows:

\subsection{Family I $\left(\beta,_{r} \neq 0\right)$}

$\mathcal{M},{ }_{r}^{2}+T,{ }_{r}^{2} \neq 0$, and $S=S(t, r) .{ }^{5}$ Moreover:

$$
e^{\nu}=\frac{f(r)}{a(r)\left(x^{2}+y^{2}\right)+2 b(r) x+2 c(r) y+d(r)}
$$

$f$ is arbitrary and:

$$
\begin{aligned}
a d-b^{2}-c^{2} & =\frac{\epsilon}{4}, \quad \epsilon=\{0, \pm 1\} ; \\
W^{2} & =\left(\epsilon-\kappa f^{2}\right)^{-1} \\
\beta_{+} & =-\frac{\kappa f \mathcal{M},_{r}}{3 \mathcal{M}}, \quad \beta_{-}=\frac{f T,_{r}}{6 \mathcal{M}} ; \\
A & =f \nu_{, r}-\kappa \beta_{+} .
\end{aligned}
$$

\footnotetext{
${ }^{4}$ See [49] for their analytical expression in case there is no cosmological constant, and [52] for the case $\kappa=0$ and $\Lambda \neq 0$.

${ }^{5} T(r)$ is the, position dependent, time of the big bang, see [49].
} 
Functions $f, a, b, c, d, \mathcal{M}$ and $T$ are arbitrary, but one should remember that there is the freedom to make a change of coordinates of the form $r \mapsto g(r)$, so, keeping in mind condition (2.8), there are actually five arbitrary functions (see also [47]).

\section{$2.2 \quad$ Family II $\left(\beta,{ }_{r}=0\right)$}

$\mathcal{M}$ and $T$ are constants, $S=S(t)$ and $W=1$. Moreover:

$$
\begin{gathered}
e^{\nu}=\frac{1}{1+\frac{\kappa}{4}\left(x^{2}+y^{2}\right)} \quad \kappa=\{0 ; \pm 1\} \\
A= \begin{cases}e^{\nu}\left\{a(r)\left[1-\frac{\kappa}{4}\left(x^{2}+y^{2}\right)\right]+b(r) x+c(r) y\right\}-\kappa \beta_{+}(r) & \kappa= \pm 1 ; \\
a(r)+b(r) x+c(r) y+\frac{\beta_{+}(r)}{2}\left(x^{2}+y^{2}\right) & \kappa=0 ;\end{cases}
\end{gathered}
$$

$a, b, c$ are arbitrary. In [52] it is shown that for the case $\kappa=0$ and $\Lambda \neq 0$, we have:

$$
A=a(r)+b(r) x+c(r) y+\frac{\beta_{+}}{4}\left(x^{2}+y^{2}\right) \sqrt[3]{4 \mathcal{M}^{2} \Lambda}
$$

Function $a, b, c, \beta_{ \pm}$are arbitrary, but, with the coordinate freedom reported above, one can see that there are actually four arbitrary functions (see [47]).

\subsection{Density contrast and shear}

In both families the density is given by:

$$
\rho(t, x, y, r)=\frac{6 \mathcal{M}}{S^{3}} \frac{A}{H}=\frac{6 \mathcal{M}}{S^{3}}\left(1+\frac{F}{H}\right) .
$$

The density contrast is given by:

$$
\delta:=\frac{F}{H}
$$

for both families (but see [55]). Since the density is non negative, one needs $\delta>-1$.

The non-null components of the observer's rate of shear tensor are given by [53]:

$$
\begin{aligned}
\sigma_{r}^{r} & =\frac{2}{3} \frac{\dot{\delta}}{1+\delta}:=\sigma ; \\
\sigma_{x}^{x} & =\sigma_{y}^{y}=-\frac{1}{2} \sigma,
\end{aligned}
$$

where we defined $\sigma:=\frac{2}{3} \frac{\dot{\delta}}{1+\delta}$ the first eigenvalue of the rate of the shear tensor.

We emphasize that it depends on $\dot{\delta}$, the rate of structure growth. 


\subsection{Cosmographic parameters}

We define the Hubble parameter in the usual fashion as: ${ }^{6}$

$$
\begin{aligned}
\mathcal{H} & =\frac{\dot{S}}{S}=\sqrt{\frac{2 \mathcal{M}}{S^{3}}+\frac{\Lambda}{3}-\frac{\kappa}{S^{2}}} \\
& =\mathcal{H}_{0} \sqrt{\frac{\Omega_{m}}{S^{3}}+\Omega_{\Lambda}+\frac{\Omega_{\kappa}}{S^{2}}}
\end{aligned}
$$

where we defined:

$$
\Omega_{m}=\frac{6 \mathcal{M}}{3 \mathcal{H}_{O}^{2}}, \quad \Omega_{\Lambda}=\frac{\Lambda}{3 \mathcal{H}_{O}^{2}}, \quad \Omega_{\kappa}=-\frac{\kappa}{\mathcal{H}_{O}^{2}} .
$$

It is easy to prove that we have also the usual relation for the present time cosmological parameters:

$$
1=\Omega_{m}+\Omega_{\Lambda}+\Omega_{\kappa}
$$

It is not difficult to prove that, if we define the deceleration parameter associated to $\mathcal{H}$ as:

$$
q=-\frac{\ddot{S}}{S} \frac{1}{\mathcal{H}^{2}}
$$

we have, at the present epoch $(S \equiv 1)$ :

$$
q_{O}=\frac{\Omega_{m}}{2}-\Omega_{\Lambda}=\frac{1-\Omega_{k}}{2}-\frac{3}{2} \Omega_{\Lambda} .
$$

One can also define a jerk parameter:

$$
j=\frac{1}{\mathcal{H}^{3}} \frac{\dot{\tilde{S}}}{S}
$$

It is easy to show that, at present time (with $S=1$ ), we have $j_{O} \equiv 1$.

\subsection{Null geodesics and redshift}

Null geodesics are defined by the condition:

$$
\begin{aligned}
& 0=k^{\alpha} k_{\alpha} \\
& 0=\left(k^{0}\right)^{2}-e^{2 \nu} S^{2}\left[\left(k^{1}\right)^{2}+\left(k^{2}\right)^{2}\right]-(H S)^{2}\left(k^{3}\right)^{2}
\end{aligned}
$$

where $k^{\alpha}=\frac{d x^{\alpha}}{d \lambda}$, and $\lambda$ is an affine parameter: $\lambda_{O}$ is the position of the observer, $\lambda_{S}$ is the position of the source. The geodesics equation is:

$$
\frac{d k^{\alpha}}{d \lambda}=-\Gamma_{\beta \gamma}^{\alpha} k^{\beta} k^{\gamma}
$$

We give their expression in appendix $\mathrm{C}$ (the Christoffel symbols needed for the calculation are reported in appendix B).

The redshift $z$ is defined as:

$$
1+z=\frac{k^{0}(\lambda)}{k^{0}\left(\lambda_{O}\right)} .
$$

\footnotetext{
${ }^{6}$ This is valid for both families, but we remind that $\mathcal{H}=\mathcal{H}(r, t)$ for Family I and $\mathcal{H}=\mathcal{H}(t)$ for Family II.
} 


\section{Luminosity distance}

Since there is no evidence for local structure growth, we make the assumption that $\dot{\delta}(0)=0$; from equation (2.16), this means that

$$
\sigma(0)=0 \text {. }
$$

We base our work on Sachs equations [57], and calculate angular diameter distance $D_{A}$ from [58]:

$$
\left\{\begin{array}{l}
\frac{d^{2} D_{A}}{d \lambda^{2}}=-\left(|\Sigma|^{2}+\frac{1}{2} R_{\alpha \beta} k^{\alpha} k^{\beta}\right) D_{A} \\
\frac{d \Sigma}{d \lambda}+2\left(\frac{d}{d \lambda} \ln D_{A}\right) \Sigma=C_{\alpha \beta \mu \nu} \epsilon^{* \alpha} k^{\beta} \epsilon^{* \mu} k^{\nu}
\end{array}\right.
$$

where: $\Sigma$ is the shear of the light bundle, $R_{\alpha \beta}$ is the Ricci tensor, $C_{\alpha \beta \mu \nu}$ is the Weyl tensor, $k^{\mu}$ is the null vector, $\epsilon^{\mu}$ is space-like and orthogonal to $k^{\mu}$, and, finally, $\lambda$ is the affine parameter.

In terms of redshift, we have, for the first equation:

$$
\left(\frac{d z}{d \lambda}\right)^{2} \frac{d^{2} D_{A}}{d z^{2}}+\left(\frac{d^{2} z}{d \lambda^{2}}\right) \frac{d D_{A}}{d z}=-\left(|\Sigma|^{2}+\frac{1}{2} R_{\alpha \beta} k^{\alpha} k^{\beta}\right) D_{A}
$$

The initial conditions are [59]:

$$
D_{A}(0)=0,\left.\quad \frac{d D_{A}}{d z}\right|_{O}=\frac{1}{\mathcal{H}_{O}} .
$$

We also impose $\Sigma(0)=0$ (see [52]).

The term depending on Ricci tensor, is given by (we use the definition of the redshift, eqn. (2.26)):

$$
\frac{1}{2} R_{\alpha \beta} k^{\alpha} k^{\beta}=\frac{3}{2} \Omega_{m}(1+\delta)\left(\mathcal{H} k^{0}\right)_{O}^{2} \frac{(1+z)^{2}}{S^{3}} .
$$

The coefficients in front of derivatives can be calculated from the definition of redshift given in equation (2.26) and the geodetic equations given in appendix C:

$$
\begin{aligned}
\left(\frac{d z}{d \lambda}\right)^{2} & =\left(\mathcal{H} k^{0}(0)\right)^{2}(1+z)^{4}-2\left(\mathcal{H} k^{0}(0)\right)\left(\frac{3}{2} \frac{\sigma}{k^{0}(0)}\right)\left(k^{3} H W S\right)^{2}+ \\
& +\left(\frac{3}{2} \frac{\sigma}{k^{0}(0)}\right)^{2}\left(k^{3} H W S\right)^{4}, \\
\frac{d^{2} z}{d \lambda^{2}} & =-\frac{d \mathcal{H}}{d \lambda} k^{0}(0)(1+z)^{2}-2(1+z)\left(\mathcal{H} k^{0}(0)\right) \frac{d z}{d \lambda}+ \\
& +\left(\frac{3}{2} \frac{d \sigma}{d z} \frac{1}{k^{0}(0)}\right) \frac{d z}{d \lambda}\left(k^{3} H W S\right)^{2}+\left(\frac{3}{2} \frac{\sigma}{k^{0}(0)}\right) \frac{d}{d \lambda}\left(k^{3} H W S\right)^{2},
\end{aligned}
$$

where we remind that $W \equiv 1$ for Family II models and, using the definition of $k^{\alpha}$ in section given $2.5:^{7}$

$$
\frac{d \mathcal{H}}{d \lambda}= \begin{cases}k^{0} \dot{\mathcal{H}}+k^{3} \frac{\partial \mathcal{H}}{\partial r} & \text { Fam I } \\ k^{0} \dot{\mathcal{H}} & \text { Fam II. }\end{cases}
$$

\footnotetext{
${ }^{7}$ In the following a dot 'stands for a time derivative, while a prime' stands for a redshift derivative.
} 
It is easy to show that in the first line of equations (3.8), one has:

$$
\frac{1}{\mathcal{H}_{O}}\left(\frac{\partial \mathcal{H}}{\partial r}\right)_{O}=\left.\frac{1}{\mathcal{H}_{O}^{2}} \frac{\partial \mathcal{M}}{\partial r}\right|_{O}-\left.\frac{\partial S}{\partial r}\right|_{O}\left(q_{O}+1\right) ;
$$

so, in order to have a definite expression for this term, one has to give an expression at least for the arbitrary functions $\mathcal{M}(r)$ and $T(r)$, since these are the arbitrary functions defining $S$ (see section 2 and [49]).

We are interested in the effects of the inhomogeneities on the acceleration of the expansion and on the possible effects on the jerk parameter (which contains information on the dark energy equation of state). Therefore we only need the Taylor expansion of $D_{A}(z)$ up to the third order:

$$
D_{A}(z)=\frac{z}{\mathcal{H}_{O}}+\frac{z^{2}}{2 \mathcal{H}_{O}} A+\frac{z^{3}}{6 \mathcal{H}_{O}} B+O\left(z^{4}\right) .
$$

We susbstitute it in (3.3) with Taylor expanded coefficients (3.6) and (3.7), in order to get $A$ e $B$.

In the following two subsection we will give an expression for $A$ and $B$ for the two families.

\subsection{Family I Szekeres cosmological models}

Since our aim here is not to construct a phenomenological model, we will not try to give a definite expression for the arbitrary functions, instead we will stop at the second order for Family I and study the effects of the inhomogenehities only on the deceleration parameter. ${ }^{8}$

In Family II we do not need to specify a model and we will go up to the third order and study the effects of inhomogeneities on the jerk parameter.

It is easy to get:

$$
A=-3-q_{O}+\left(\left.\frac{3}{2} \frac{\sigma^{\prime}}{\mathcal{H}}\right|_{O}\right)\left(\frac{k^{3}}{k^{0}}\right)_{O}^{2}+\left(\left.\frac{1}{\mathcal{H}_{O}} \frac{\partial \mathcal{H}}{\partial r}\right|_{O}\right)\left(\frac{k^{3}}{k^{0}}\right)_{O},
$$

where $q_{O}$ is given in (2.22).

Luminosity distance is given by:

$$
\begin{aligned}
D_{L}(z) & =(1+z)^{2} D_{A}(z) \\
& =\frac{D_{L}^{(1)}}{\mathcal{H}_{0}} z+\frac{D_{L}^{(2) I}}{2 \mathcal{H}_{0}} z^{2}+O\left(z^{3}\right),
\end{aligned}
$$

where:

$$
\begin{aligned}
D_{L}^{(1)}=1 \\
D_{L}^{(2) I}=1-q_{O}+\left(\left.\frac{3}{2} \frac{\sigma^{\prime}}{\mathcal{H}}\right|_{O}\right)\left(\frac{k^{3}}{k^{0}}\right)_{O}^{2}+\left(\left.\frac{1}{\mathcal{H}_{O}} \frac{\partial \mathcal{H}}{\partial r}\right|_{O}\right)\left(\frac{k^{3}}{k^{0}}\right)_{O}= \\
=1-q_{\text {eff }}^{I},
\end{aligned}
$$

${ }^{8}$ Without a definite expression for the arbitrary functions, we find a proliferation of terms like $\frac{S, r}{S}$ and $\frac{\dot{S}, r}{S}$ difficult to manage. 
where we defined the effective deceleration parameter for Family I:

$$
q_{\text {eff }}^{I}:=q_{O}-\left(\left.\frac{3}{2} \frac{\sigma^{\prime}}{\mathcal{H}}\right|_{O}\right)\left(\frac{k^{3}}{k^{0}}\right)_{O}^{2}-\left(\left.\frac{1}{\mathcal{H}_{O}} \frac{\partial \mathcal{H}}{\partial r}\right|_{O}\right)\left(\frac{k^{3}}{k^{0}}\right)_{O} .
$$

It depends on the radial derivative of the Hubble parameter given in (3.9) (the cosmic variance problem), on the direction of observation (see section 4) and on $\sigma^{\prime}(0)$, which, we remind, is linked to the inhomogeneities growth (see equation (2.16)).

\subsection{Family II Szekeres cosmological models}

As already said, in the case of Family II cosmological models, we can easily Taylor expand the angular diameter distance up to the third order without specifying any particular model.

Using equations (C.1), (C.4), we find:

$$
A=-3-q_{O}+\left(\left.\frac{3}{2} \frac{\sigma^{\prime}}{\mathcal{H}}\right|_{O}\right)\left(\frac{k^{3}}{k^{0}}\right)_{O}^{2},
$$

for the second order, while for the third order, we have:

$$
\begin{aligned}
B & =3 A^{2}-j_{O}-10\left[q_{O}-\left(\frac{3}{2} \frac{\sigma^{\prime}}{\mathcal{H}}\right)\left(\frac{k^{3}}{k^{0}}\right)^{2}\right]_{O}-15+2\left(\left.\frac{3}{2} \frac{\sigma^{\prime}}{\mathcal{H}}\right|_{O}\right)\left(\frac{k^{3}}{k^{0}}\right)_{O}^{2}+ \\
& +4\left(\left.\frac{3}{2} \frac{\sigma^{\prime}}{\mathcal{H}}\right|_{O}\right)\left(\frac{k^{3}}{k^{0}}\right)_{O}^{2}\left[\frac{k^{1}}{k^{0}} \frac{\partial}{\partial x} \ln H+\frac{k^{2}}{k^{0}} \frac{\partial}{\partial y} \ln H\right]_{O}+ \\
& -\frac{3}{2} \Omega_{m}(1+\delta(0)) .
\end{aligned}
$$

Luminosity distance is given, as usual, by $D_{L}(z)=(1+z)^{2} D_{A}(z)$, and we find:

$$
\begin{aligned}
D_{L}(z) & =\frac{z}{\mathcal{H}_{O}}\left(1+2 z+z^{2}\right)\left(1+\frac{z}{2} A+\frac{z^{2}}{6} B+O\left(z^{3}\right)\right)= \\
& =\frac{z}{\mathcal{H}_{O}}+\frac{z^{2}}{2 \mathcal{H}_{O}}(4+A)+\frac{z^{3}}{6 \mathcal{H}_{O}}(B+6 A+6)+O\left(z^{4}\right)= \\
& =\frac{D_{L}^{(1)}}{\mathcal{H}_{O}} z+\frac{D_{L}^{(2) I I}}{2 \mathcal{H}_{O}} z^{2}+\frac{D_{L}^{(3) I I}}{6 \mathcal{H}_{O}} z^{3}+O\left(z^{4}\right),
\end{aligned}
$$

where:

$$
\begin{gathered}
D_{L}^{(1)}=1 ; \\
D_{L}^{(2) I I}=1-q_{O}+\left(\left.\frac{3}{2} \frac{\sigma^{\prime}}{\mathcal{H}}\right|_{O}\right)\left(\frac{k^{3}}{k^{0}}\right)_{O}^{2} \\
=1-q_{\text {eff }}^{I I} ; \\
D_{L}^{(3) I I}=3\left(q_{\text {eff }}^{I I}\right)^{2}+q_{\text {eff }}^{I I}-j_{O}-\Omega_{O}+\left(\left.\frac{3}{2} \frac{\sigma^{\prime}}{\mathcal{H}}\right|_{O}\right)+\left(\left.\frac{3}{2} \frac{\sigma^{\prime}}{\mathcal{H}}\right|_{O}\right)\left(\frac{k^{3}}{k^{0}}\right)_{O}^{2}+ \\
+4\left(\left.\frac{3}{2} \frac{\sigma^{\prime}}{\mathcal{H}}\right|_{O}\right)\left(\frac{k^{3}}{k^{0}}\right)_{O}^{2}\left[\frac{k^{1}}{k^{0}} \frac{\partial}{\partial x} \ln H+\frac{k^{2}}{k^{0}} \frac{\partial}{\partial y} \ln H\right]_{O} .
\end{gathered}
$$


In the previous equation, we defined the parameter $\Omega_{O}=1-\Omega_{k}$ (see also [6]), and the effective deceleration parameter for Family II models, $q_{e f f}^{I I}$ :

$$
q_{\text {eff }}^{I I}:=q_{O}-\left(\left.\frac{3}{2} \frac{\sigma^{\prime}}{\mathcal{H}}\right|_{O}\right)\left(\frac{k^{3}}{k^{0}}\right)_{O}^{2} .
$$

\section{Angular dependence}

The terms $\left.\left(\frac{k^{i}}{k^{0}}\right)\right|_{O}$ that appear in the previous equations are direction dependent.

Following, for example, [52], we define, at the observer position, $\alpha$, the angle formed by the $k^{\mu}$ with the $r$ axis, and $\beta$, the angle formed with the $r-x$ plane; in this way we have:

$$
\begin{aligned}
& k^{1}(0)=k^{0}(0) \cos \beta \sin \alpha ; \\
& k^{2}(0)=k^{0}(0) \sin \beta \sin \alpha ; \\
& k^{3}(0)=k^{0}(0) \cos \alpha .
\end{aligned}
$$

\subsection{The effective deceleration parameters}

The usual expression for the second order of the luminosity distance is [56]:

$$
D_{L}^{(2)}=1-q_{o b s},
$$

where $q_{o b s}$ is the observed deceleration parameter.

We defined the deceleration parameters in equation (3.15) for Family I and in equation (3.20) for Family II. Using equations (4.1)-(4.3), we rewrite them respectively as:

$$
\begin{aligned}
q_{\text {eff }}^{I} & =q_{O}-\left(\left.\frac{1}{\mathcal{H}_{O}} \frac{\partial \mathcal{H}}{\partial r}\right|_{O}\right) \cos \alpha-\left(\left.\frac{3}{2} \frac{\sigma^{\prime}}{\mathcal{H}}\right|_{O}\right) \cos ^{2} \alpha= \\
& =q_{O}-\left(\left.\frac{1}{\mathcal{H}_{O}} \frac{\partial \mathcal{H}}{\partial r}\right|_{O}\right) \cos \alpha+\frac{3}{2} \delta(0) \Omega_{M} \cos ^{2} \alpha ; \\
q_{e f f}^{I I} & =q_{O}-\left(\left.\frac{3}{2} \frac{\sigma^{\prime}}{\mathcal{H}}\right|_{O}\right) \cos ^{2} \alpha= \\
& =q_{O}+\frac{3}{2} \Omega_{M} \delta(0) \cos ^{2} \alpha .
\end{aligned}
$$

where in (4.6) and (4.8), we used the relation:

$$
\left.\frac{\sigma^{\prime}}{\mathcal{H}}\right|_{O}=-\delta(0) \Omega_{m}
$$

derived in the appendix A. 
We notice how in both cases the presence of a local inhomogeneity gives rise to an angular dependence of the deceleration parameter; in Family I we see that the dependence of the Hubble parameter on the radial coordinate, gives rise to an additional angular dependence. We notice further that only the angle $\alpha$, formed by the direction of observation and the $r$ axis, is involved in the expression.

The dependence of the Hubble parameter on the radial coordinate is a form of cosmic variance: different observers at different positions measure different value for the cosmographic parameter. As already argued by [40] this might mitigate the tension between the local measurement of $\mathcal{H}$ and the ones coming from $\mathrm{BAO}$ and $\mathrm{CMB}^{9}$.

Averaging over directions gives:

$$
\begin{aligned}
\left\langle q_{\text {eff }}^{I}\right\rangle=\left\langle q_{\text {eff }}^{I I}\right\rangle & =\frac{1-\Omega_{k}}{2}-\frac{3}{2}\left[\Omega_{\Lambda}+\left.\frac{1}{3} \frac{\sigma^{\prime}}{\mathcal{H}}\right|_{O}\right]= \\
& =\frac{1-\Omega_{k}}{2}(1+\delta(0))-\frac{3}{2} \Omega_{\Lambda}\left(1+\frac{\delta(0)}{3}\right) .
\end{aligned}
$$

From the first equality we notice that if $\sigma^{\prime}(0)>0$ (and therefore $\delta(0)<0$, from (A.6)), less dark energy is needed in order to explain acceleration.

\subsection{The effective jerk parameter $j_{e f f}$}

The usual expression for $D_{L}^{(3)}$ in $\Lambda$ CDM models (for arbitrary values of $\kappa$ ) is given by (see for example $[6,60])$ :

$$
D_{L}^{(3)}=3 q_{o b s}^{2}+q_{o b s}-\Omega_{O}-j_{o b s}
$$

where one expects $j_{\text {obs }} \equiv 1[10,21,60]$.

In our case we have, for Family II's third order:

$$
\begin{aligned}
D_{L}^{(3) I I} & =3\left(q_{\text {eff }}^{I I}\right)^{2}+q_{\text {eff }}^{I I}-j_{O}-\Omega_{O}+\left(\left.\frac{3}{2} \frac{\sigma^{\prime}}{\mathcal{H}}\right|_{O}\right)+\left(\left.\frac{3}{2} \frac{\sigma^{\prime}}{\mathcal{H}}\right|_{O}\right) \cos ^{2} \alpha+ \\
& +4\left(\left.\frac{3}{2} \frac{\sigma^{\prime}}{\mathcal{H}}\right|_{O}\right) \cos ^{2} \alpha\left[\cos \beta \sin \alpha \frac{\partial}{\partial x} \ln H+\sin \alpha \sin \beta \frac{\partial}{\partial y} \ln H\right]_{O}
\end{aligned}
$$

We can read off the expression for the effective jerk parameter by comparing the previous equation with (4.11):

$$
\begin{aligned}
j_{\text {eff }} & =j_{O}-\left(\left.\frac{3}{2} \frac{\sigma^{\prime}}{\mathcal{H}}\right|_{O}\right)-\left(\left.\frac{3}{2} \frac{\sigma^{\prime}}{\mathcal{H}}\right|_{O}\right) \cos ^{2} \alpha+ \\
& -4\left(\left.\frac{3}{2} \frac{\sigma^{\prime}}{\mathcal{H}}\right|_{O}\right) \cos ^{2} \alpha\left[\cos \beta \sin \alpha \frac{\partial}{\partial x} \ln H+\sin \alpha \sin \beta \frac{\partial}{\partial y} \ln H\right]_{O}= \\
& =j_{O}+\frac{3}{2} \Omega_{M} \delta(0)\left(1+\cos ^{2} \alpha\right)+ \\
& +4\left(\frac{3}{2} \Omega_{M} \delta(0)\right) \cos ^{2} \alpha\left[\cos \beta \sin \alpha \frac{\partial}{\partial x} \ln H+\sin \alpha \sin \beta \frac{\partial}{\partial y} \ln H\right]_{O}
\end{aligned}
$$

where, as we said earlier, $j_{O} \equiv 1$ and where we used equation (A.6) in the second equatility.

The presence of the inhomogeneity gives rise to a change of the value of the jerk from the $\Lambda \mathrm{CDM}$ one; moreover, in this case we see that there is a dependence on both angles $\alpha$

\footnotetext{
${ }^{9}$ See [41] and [42] for older works on the same topic.
} 
and $\beta$ coming form the $x$ - and $y$-derivatives of $H$. From its definition, it is easy to see that those are the derivatives of the density contrast:

$$
\frac{1}{H} \frac{\partial H}{\partial x}=-\frac{1}{\delta} \frac{\partial \delta}{\partial x} \quad \frac{1}{H} \frac{\partial H}{\partial y}=-\frac{1}{\delta} \frac{\partial \delta}{\partial y} .
$$

Substituting in (4.13), we have:

$$
\begin{aligned}
j_{e f f} & =j_{O}+\frac{3}{2} \Omega_{M} \delta(0)\left(1+\cos ^{2} \alpha\right)+ \\
& -6 \Omega_{M} \cos ^{2} \alpha\left[\cos \beta \sin \alpha \frac{\partial \delta}{\partial x}+\sin \alpha \sin \beta \frac{\partial \delta}{\partial y}\right]_{O}
\end{aligned}
$$

Averaging over directions, we have:

$$
\left\langle j_{e f f}\right\rangle=j_{O}+2 \Omega_{m} \delta(0),
$$

therefore, $\delta(0)<0$ gives $\left\langle j_{\text {eff }}\right\rangle<1$, a result found by some recent works, (see [60], [6]).

\section{Comparison with observations}

As said in the introduction, the idea that inhomogeneities can have an effect on observation is not new. Many works in the literature are based on the assumption that we might live in a void (a region with $\delta<0$ ), since a void expands at a faster rate than the the background, thus mimicking the effect of a cosmological constant.

In particular, in [23] the authors use 44 close SNe Ia $\left(20-300 h^{-1} \mathrm{Mpc}\right)$ to study the monopole of peculiar velocities of the host galaxies: they find that their data are consistent with a local void with an underdensity of about $20 \%$, so $\delta(0) \approx-0.20$.

In [26], the authors fit 3-yr WMAP data and SNe Ia with the hypothesis that we live in a spherical-symmetric void described by a LTB metric: they find that they need $\delta(0) \approx-0.4$ and that the observer must be close to the center of the sphere for their results to be consistent with the CMB.

More recently, in [33] the authors use a LTB void metric to describe a local void and fit their model using $\mathrm{CMB}, \mathrm{SN}, \mathrm{BAO}$ and $\mathcal{H}_{0}$ allowing for a non null space curvature: they find that their model fits the data with a $\delta(0)=-0.65$ void that extends up to $z \approx 1$ (gigaparsec scale); they also find $\Omega_{k}=-0.2$ and that the model is consistent with the CMB dipole if the observer is displaced by about $15 \mathrm{Mpc}$ from the center.

The fact that the observer must be close to the center of the void in order to be consistent with the CMB data is a violation of the Cosmological Principle, since that position is obviously a particular one. Even if the void is non-symmetric (so that there is not an actual center), there is still a weak violation of the Principle, since usually galaxies are found in clusters not in voids, see [59].

In [35], the authors propose a way to falsify non Copernican models by constraining the parameter space of those models; they give constraints on $\delta$ and on the radius of the inhomogeneity finding that only models with $\delta(0) \approx 0$ are admissible, though many values of the radius are allowed for the Copernican principle to be verified.

As far as the jerk parameter and the dark energy equation of state ${ }^{10}$ is concerned, we see that inhomogeneities change the value from the expected $j_{o b s}=1$ : an observer might therefore interpret this effect as a change in the equation of state of dark energy with redshift.

\footnotetext{
${ }^{10}$ See $[21]$ for the link between the two.
} 
A similar result is found also in [38]: they consider a quasi-linear inhomogeneity ${ }^{11}$ in a flat $w \mathrm{CDM}$ background, with a constant $w$; they test their model with $\mathrm{SNe}$, CMB and local measurement of the Hubble parameter and find $\delta(0)=0.1 \div 0.15$ and that $w=-1$ is excluded at $95 \%$ of confidence level. They also find a degeneracy between $w$ and $\delta(0)$ and argue that even if $w$ is constant in the background, the effect of the inhomogeneities is to make it depend on the redshift for an observer that wanted to use a homogeneous model to fit the data. In [39], the authors suggest that until the value of the local over- or underdensity is known, their effect should be considered as a systematic error in $w$ measurements (systematic, because linked to the cosmic variance problem).

On this topic, see also [36], where the authors use an LTB model with a cosmological constant in order to study the effect on the dark energy equation of state; they find that an underdesity leads to an apparent phantom behavoir of the dark energy, while an over density leads to an apparent quintessence behavoir.

\subsection{Effects of angular dependency}

From the discussion in the previous section, we have that an estimate for the value of the local density contrast is $\delta(0) \approx-0.65 \div 0.15$.

One can use the value of matter density as given by the Planck collaboration [5], $\Omega_{m}=$ $0.135 \pm 0.017$, in order to estimate the corrections due to inhomogeneities: from Family II effective deceleration parameter, $q_{e f f}^{I I}$, these corrections are of percent level and should be measurable (indeed, see [66] for a claim of asymmetry in supernova data); in case of Family I, there is also the correction coming from the Hubble parameter radial derivative which amuonts to about $1 \%$.

We can say nothing about the jerk parameter since, as far as we know, there are no measure for the density gradient, but the second term in equation (4.15) gives again a correction at percent level.

\section{Conclusions}

Szekeres cosmological models are inhomogeneus and anisotropic solutions of the Einstein's Field equations with irrotational dust and a cosmological constant as a source.

Starting from the Sachs equations, we have calculated the Taylor expansion of the Szekeres luminosity distance for both Family I and Family II allowing for the existence of a cosmological constant. Relying on observations, we make the hypotesis that there is no structure growth at the observer position: $\dot{\delta}(0)=0$.

Confronting our luminosity distance with the usual expansion of the $\Lambda$ CDM luminosity distance, we have found corrections proportional to $\sigma^{\prime}(0)$ that are also direction dependent. $\sigma$ is the eigenvalue of the observer's rate of the shear tensor $\sigma=\sigma_{r}^{r}$ and is linked to the evolution of the density contrast (see equation (2.16)). Therefore, inhomogeneities and local structure evolution affect the way an observer sees the expansion.

In Family I models, at second order, there is also a correction proportional to $\left.\frac{\partial \mathcal{H}}{\partial r}\right|_{O}$, a consequence of the cosmic variance. This could give an explanation to the tension between local direct extimates of $\mathcal{H}_{O}$ and indirect ones from BAO and CMB (see [40]-[42]).

\footnotetext{
${ }^{11}$ This inhomogeneity is constructed using the model developed in [37].
} 
Using data from the literature, we find that inhomogeneities could give corrections of percent level on the deceleration parameter: direction dependence should therefore be observable. In the case of the effective jerk parameter, a measure of the density gradient is needed in order to give an estimate of the correction.

\section{A Useful relations}

In this appendix we give a derivation of equation (4.9), which relates $\sigma^{\prime}(0)$ and the density contrast $\delta(0)$ at the observer's position, and we give an analogue expression also for $\sigma^{\prime \prime}(0)$.

Since, in Family II models, we can impose that $H_{O}=S_{O}=1$, we have:

$$
\begin{gathered}
\frac{3}{2} \sigma(0)=\left.\frac{\dot{F}}{H}\right|_{O}=\left.\dot{F}\right|_{O}=0 ; \\
\delta(0)=\left.\frac{F}{H}\right|_{O}=\left.F\right|_{O} .
\end{gathered}
$$

Since for Family I, we have $(H W)_{O}=1$, we have, in this case:

$$
\delta(0)=\left.\frac{F}{H}\right|_{O}=(F W)_{O}
$$

Using the definition of redshift and of $k^{0}$ given in 2.5, the first derivative of the $\sigma$ with respect to the redshift calculated at the position of the observer is:

$$
\begin{aligned}
\frac{3}{2} \sigma^{\prime}(0) & =\left.\frac{3}{2} \frac{d \sigma}{d z}\right|_{O}= \\
& =\left.\frac{3}{2} \frac{d \lambda}{d z} \frac{d t}{d \lambda} \frac{d \sigma}{d t}\right|_{O}= \\
& =-\left.\frac{3}{2} \frac{1}{\mathcal{H}_{O}} \frac{d \sigma}{d t}\right|_{O}= \\
& =-\frac{1}{\mathcal{H}_{O}}\left[\frac{\ddot{F}}{H}+\left(\frac{\dot{F}}{H}\right)^{2}\right]_{O},
\end{aligned}
$$

where in the third line we used equation (2.26) and the definition of $k^{0}$ given in 2.5. In the last line, the second term is zero because of equation (A.1). We now use the Raychaudhury equation (2.6), so we get:

$$
\begin{aligned}
\frac{3}{2} \sigma^{\prime}(0) & =-\left.\frac{1}{\mathcal{H}_{O}} \ddot{F}\right|_{O}=-\frac{1}{\mathcal{H}_{O}}\left[-2 \mathcal{H} \dot{F}+\frac{3 \mathcal{M}}{S^{3}} F\right]_{O}= \\
& =-\frac{1}{\mathcal{H}_{O}}\left[\frac{3}{2} \Omega_{m} \mathcal{H}_{O}^{2} \delta(0)\right]
\end{aligned}
$$


In the second line we used again (A.1) and (A.2) and also the definition of $\Omega_{m}$. Simplifying, we get:

$$
\sigma^{\prime}(0)=-\Omega_{m} \delta(0) \mathcal{H}_{O} .
$$

which is the expression given in (4.9).

On the same line, we find, for Family II:

$$
\begin{aligned}
\frac{3}{2} \sigma^{\prime \prime}(0) & =-\left(q_{O}-3\right)\left(\left.\frac{3}{2} \frac{\sigma^{\prime}}{\mathcal{H}}\right|_{O}\right)+\left(\left.\frac{3}{2} \frac{\sigma^{\prime}}{\mathcal{H}}\right|_{O}\right)^{2}\left(\frac{k^{3}}{k^{0}}\right)_{O}^{2}= \\
& =\left(q_{O}-3\right)\left(\frac{3}{2} \Omega_{m} \mathcal{H}_{O} \delta(0)\right)+\left(\frac{3}{2} \Omega_{m} \mathcal{H}_{O} \delta(0)\right)^{2}\left(\frac{k^{3}}{k^{0}}\right)_{O}^{2}
\end{aligned}
$$

where in the second line we used (A.6).

We also report, without proving them, two relations involving the first and second time derivatives of the Hubble parameter and the deceleration and the jerk parameters needed in our calculations: ${ }^{12}$

$$
\begin{aligned}
& \dot{\mathcal{H}}=-\mathcal{H}^{2}(1+q) ; \\
& \ddot{\mathcal{H}}=\mathcal{H}^{3}(j+3 q+2) .
\end{aligned}
$$

\section{B Christoffel symbols}

In this appendix we report the non-null Christoffel symbols for the Goode-Wainwright representation for both families needed for calculating the geodesics.

\section{Family I}

$$
\begin{gathered}
\Gamma_{11}^{0}=\Gamma_{22}^{0}=\mathcal{H}\left(S^{2} e^{2 \nu}\right) \\
\Gamma_{33}^{0}=\left(\frac{\dot{H}}{H}+\frac{\dot{S}}{S}\right)\left(S^{2} W^{2} H^{2}\right) \\
\Gamma_{10}^{1}=\Gamma_{20}^{2}=\mathcal{H} \\
\Gamma_{30}^{3}=\frac{\dot{H}}{H}+\frac{\dot{S}}{S} \\
\Gamma_{12}^{1}=\Gamma_{22}^{2}=-\Gamma_{11}^{2}=\frac{\partial}{\partial y} \ln \left(e^{\nu} S\right) \\
\Gamma_{13}^{1}=\Gamma_{23}^{2}=\frac{\partial}{\partial r} \ln \left(e^{\nu} S\right) \\
\Gamma_{11}^{1}=\Gamma_{21}^{2}=-\Gamma_{22}^{1}=\frac{\partial}{\partial x} \ln \left(e^{\nu} S\right) \\
\Gamma_{33}^{1}=-\frac{W^{2} H^{2}}{e^{2 \nu}} \frac{\partial}{\partial x} \ln (H W S) \\
\Gamma_{33}^{2}=-\frac{H^{2} W^{2}}{e^{2 \nu}} \frac{\partial}{\partial y} \ln (H)
\end{gathered}
$$

\footnotetext{
${ }^{12}$ See also [6], [60] and [62].
} 


$$
\begin{gathered}
\Gamma_{11}^{3}=\Gamma_{22}^{3}=-\frac{e^{2 \nu}}{H^{2} W^{2}} \frac{\partial}{\partial r} \ln \left(S e^{\nu}\right) \\
\Gamma_{31}^{3}=\frac{\partial}{\partial x} \ln (H) \\
\Gamma_{32}^{3}=\frac{\partial}{\partial y} \ln (H) \\
\Gamma_{33}^{3}=\frac{\partial}{\partial r} \ln (H W S)
\end{gathered}
$$

\section{Family II}

$$
\begin{gathered}
\Gamma_{11}^{0}=\Gamma_{22}^{0}=\mathcal{H}\left(S^{2} e^{2 \nu}\right) ; \\
\Gamma_{33}^{0}=(H S)^{2}\left(\frac{\dot{H}}{H}+\frac{\dot{S}}{S}\right) ; \\
\Gamma_{03}^{3}=\left(\frac{\dot{H}}{H}+\frac{\dot{S}}{S}\right) ; \\
\Gamma_{01}^{1}=\Gamma_{02}^{2}=\mathcal{H} ; \\
\Gamma_{11}^{1}=\Gamma_{12}^{2}=-\Gamma_{22}^{1}=\frac{\partial \ln \left(e^{\nu}\right)}{\partial x} ; \\
\Gamma_{22}^{2}=\Gamma_{12}^{1}=-\Gamma_{11}^{2}=\frac{\partial \ln \left(e^{\nu}\right)}{\partial y} ; \\
\Gamma_{33}^{1}=-\frac{H^{2}}{e^{2 \nu}} \frac{\partial \ln H}{\partial x} ; \\
\Gamma_{33}^{2}=-\frac{H^{2}}{e^{2 \nu}} \frac{\partial \ln H}{\partial y} ; \\
\Gamma_{13}^{3}=\frac{\partial \ln H}{\partial x} ; \\
\Gamma_{23}^{3}=\frac{\partial \ln H}{\partial y} ; \\
\Gamma_{33}^{3}=\frac{\partial \ln H}{\partial r} .
\end{gathered}
$$




\section{Null geodesics and redshift}

In this appendix we report null geodesics for both families calculated using Christoffel symbols given in the previous appendix and the null condition given in section 2.5.

\section{Family I}

$$
\begin{aligned}
\frac{d k^{0}}{d \lambda} & =-\mathcal{H}\left(k^{0}\right)^{2}+\frac{3}{2} \sigma\left(H W S k^{3}\right)^{2} \\
\frac{d}{d \lambda}\left[k^{1}\left(e^{\nu} S\right)^{2}\right] & =\frac{1}{2} \frac{\partial}{\partial x}\left(e^{2 \nu} S^{2}\right)\left[\left(k^{0}\right)^{2}-(H W S)^{2}\left(k^{3}\right)^{2}\right]-e^{2 \nu} S^{2}\left(k^{3}\right)^{2} \frac{\partial}{\partial x} \ln (H) ; \\
\frac{d}{d \lambda}\left[k^{2}\left(e^{\nu} S\right)^{2}\right] & =\frac{1}{2} \frac{\partial}{\partial y}\left(e^{2 \nu} S^{2}\right)\left[\left(k^{0}\right)^{2}-(H W S)^{2}\left(k^{3}\right)^{2}\right]-e^{2 \nu} S^{2}\left(k^{3}\right)^{2} \frac{\partial}{\partial y} \ln (H) ; \\
\frac{d}{d \lambda}\left[k^{3}(H S W)^{2}\right] & =(H W S)^{2}\left[\left(k^{0}\right)^{2}-\left(k^{3}\right)^{2}(H W S)^{2}\right] \frac{\partial}{\partial r} \ln \left[\left(e^{\nu} S\right)\right]+ \\
& +\frac{1}{2} \frac{\partial}{\partial r}[H W S]^{2}\left(k^{3}\right)^{2} .
\end{aligned}
$$

\section{Family II}

$$
\begin{aligned}
\frac{d k^{0}}{d \lambda} & =-\frac{d}{d \lambda} \ln (S) k^{0}+\frac{3}{2} \sigma\left(H S k^{3}\right)^{2}=-\mathcal{H}\left(k^{0}\right)^{2}+\frac{3}{2} \sigma\left(H S k^{3}\right)^{2} ; \\
\frac{d}{d \lambda}\left[k^{0} S\right] & =\frac{3}{2} \sigma\left(H S k^{3}\right)^{2} ; \\
\frac{d}{d \lambda}\left[k^{1}\left(e^{\nu} S\right)^{2}\right] & =\frac{1}{2} \frac{\partial}{\partial x}\left(e^{2 \nu} S^{2}\right)\left[\left(k^{0}\right)^{2}-(H S)^{2}\left(k^{3}\right)^{2}\right]-e^{2 \nu} S^{2}\left(k^{3}\right)^{2} \frac{\partial}{\partial x} \ln (H) ; \\
\frac{d}{d \lambda}\left[k^{2}\left(e^{\nu} S\right)^{2}\right] & =\frac{1}{2} \frac{\partial}{\partial y}\left(e^{2 \nu} S^{2}\right)\left[\left(k^{0}\right)^{2}-(H S)^{2}\left(k^{3}\right)^{2}\right]-e^{2 \nu} S^{2}\left(k^{3}\right)^{2} \frac{\partial}{\partial y} \ln (H) ; \\
\frac{d}{d \lambda}\left[k^{3}(H S)^{2}\right] & =\frac{1}{2}\left(k^{3}\right)^{2} \frac{\partial}{\partial r} H^{2} .
\end{aligned}
$$

\section{Acknowledgments}

The author wishes to thank K. Bolejko and M.N.Cèlèrier and the anonymous referee for their patience and helpful suggestions.

Note added The solutions to the Szekeres model with a csmological source were first found by J.Barrow and J.S.Schabes in [45]. I am sorry for my omission.

\section{References}

[1] A.G.Reiss et al. Observational Evidence from Supernovae for an Accelerating Universe and a Cosmological Constant, ApJ., 116, (1998) 1009-1038;

[2] S.Perlmutter et al. Measurements of Omega and Lambda from 42 High-Redshift Supernovae, ApJ. , 517, (1999) 565-586;

[3] Conley A., Sullivan M., et al., Supernova Constraints and Systematic Uncertainties from the First Three Years of the Supernova Legacy Survey, Apj., 192, (2011), 1; 
[4] N.Suzuki et al. The Hubble Space Telescope cluster supernova survey: V. Improving the dark energy constraints above $z>1$ and building an early-type-hosted supernova sample, Ap.J. 746, (2012), 85; arXiv:1105.3470 [astro-ph.CO];

[5] Planck Collaboration, Planck 2013 results. XVI. Cosmological parameters, arXiv:1303.5076 [astro-ph.CO];

[6] K. Bamba, et al, Dark enrgy cosmology: the equivalent description via different theoretical models and cosmological tests, Astrophys. Space Sci. 342 (2012), 155-228; arXiv:1205.3421 [gr-qc];

[7] L.Amendola, S.Tsujikawa Dark energy: theory and observations, Cambridge University Press (2010);

[8] E.J. Copeland, M.Sami, S.Tsujikawa Dynamics of dark energy, Int. J. Mod. Phys. D, 15, (2006), 1753;

[9] N.Straumann, Dark energy, recent developement, Mod. Phys. Lett. A, 21, (2006), 1083;

[10] S.Sahni, A.Starobinsky, Reconstructing dark energy, Int. J. Mod. Phys. D, 15, (2006), 2105;

[11] D.J.Schwartz, Accelerated expansion without dark energy, arXiv:astroph/0209584;

[12] C.Wetterich, Can structure formation influence the cosmological evolution?, Phys.Rev.D, 67, (2003), 043513; arXiv:astroph/0111166;

[13] T. Buchert, On average properties of inhomogeneous fluids in general relativity I: Dust cosmologies, Gen. Rel. Grav., 32, (2000), 105;

[14] T. Buchert, On average properties of inhomogeneous fluids in general relativity II: Perfect Fluid cosmologies, Gen. Rel. Grav., 33, (2001), 1381;

[15] C. Clarkson, O.Umeh, Is backreaction really small within concordance cosmology?, Class.Quant.Grav. 28164010 (2011); arXiv:1105.1886 [astro-ph.CO];

[16] S. Green, R.Wald, A new framework for analyzing the effects of small scale inhomogeneities in cosmology., Phys. Rev. D 83, (2011), 084020; arXiv:1011.4920 [gr-qc];

[17] B. Bochner, Cosmic acceleration from causal backreaction in a smoothly inhomogeneuos universe.; arXiv:1109.4686 [astro-ph.CO];

[18] R.J. van der Hoogen, Averaging spacetime: Where do we go from here., arXiv:1003.4020 [gr-qc];

[19] S. Räsänen, Acclelerated expansion form structures formation, JCAP, 0611, (2006), 3;

[20] S. Räsänen, Dark energy from backreaction, JCAP, 0403, (2004), 3;

[21] V. Sahni, T.D. Saini, A.A. Starobinsky, U. Allam; Statefinder - A new geometrical diagnostic of dark energy, JETP. Lett. 77 (2003), 201 arXiv: astro-ph/0201498;

[22] J.W Moffat, D.C. Takarski, Cosmological observation in local void, Ap.J. 453 17-24 (1995); arXiv:astro-ph/9407036;

[23] I.Zehavi, A.G.Riess, R.P.Kirshner, A. Dekel, A local Hubble Bubble from SNe Ia?, Ap.J., 503, (1998), 483;

[24] K.Tomita A local void and the accelerating universe, MNRAS, 326, (2001), 287;

[25] H. Alnes, M. Amarzguioui, CMB anisotropies seen by an off-center observer in a sferically simmetric inhomogeneous universe, Phys. Rev. D, 74, (2006), 103520;

[26] S.Alexander et al. Local void VS Dark Energy: Confrontation with WMAP and Type Ia Supernova, JCAP 0909, (2009), 025; arXiv:0712.0370 [astro-ph];

[27] K.Tomita, Distances and lensing in cosmological void models, Ap.J. 529, 38 (2000);

[28] M.N.Cèlèrier, Do we really see a cosmological constant in the supernovae data?, A.\& A., 353, 63 (2000); 
[29] H.Iguchi et al. Is dark energy the only solution to the apparent acceleration of the present Universe?, Prog. Theor. Phys., 108, 809 (2002);

[30] J.F. Pascual-Sánchez, Cosmic Acceleration: Inhomogeneity versus vacuum energy, Mod. Phys. Lett. A 14, 1539 (1999); arXiv:gr-qc/9905063;

[31] R. Ali Vanderveld et al., Mimiking dark energy with Lemaître-Tolman-Bondi models: Weak central singularities and critical points, Phys. Rev. D 74, 02356 (2006);

[32] K. Enqvist, Lemaître-Tolman-Bondi model and accelerating expansion, Gen. Rel. and Grav., 40, 451, (2008);

[33] B. Tirthabir et al. Testing the void against cosmological data: fitting $C M B, B A O, S N$ an $H_{0}$, JCAP 1011 (2010) 030; arXiv:1007.3065 [astro-ph.CO];

[34] V. Marra, A. Notari, Observational constrains on inhomogeneous cosmological models without dark energy, Class.Quant.Grav. 28, 164004 (2011); arXiv:1102.1015 [astro-ph.CO];

[35] W. Valkenburg, et al. Testing the Copernical principle by constraining spatial homogeneity, MNRAS, 438 L6-L10 (2014); arXiv:1209.4078 [astro-ph.CO];

[36] A.E.Romano et al., Effects of inhomogeneities on apparent cosmological observables: "fake" evolving dark energy, Eur. Phys. J. C 72, 2242 (2012); arXiv:1203.2180 [astro-ph.CO];

[37] W. Valkenburg, Perceiving the equation of state of Dark Energy while living in a Cold Spot, JCAP1201, 047, (2012), arXiv:1106.6042 [astro-ph.CO]

[38] V. Marra, et al. Uncertainty on w from large-scale structure, MNRAS 431, 1891, (2013); arXiv:1203.2180 [astro-ph.Co];

[39] W. Valkenburg, et al. Intrinsic uncertainty on the nature of dark energy, Phys.Dark Univ. 2, 219-223 (2013); arXiv:1302.6588 [astro-ph.CO];

[40] V. Marra, et al. Cosmic variance and the measurement of the local Hubble parameter, Phys.Rev.Lett. 110241305 (2013); arXiv:1303.3121 [astro-ph.CO];

[41] E. L. Turner, et al., The relation of local measurement of Hubble's constant to its global value, Ap.J. 103, 1492 (1992);

[42] R. Giovanelli, et al. No Hubble Bubble in the Local Universe, Ap.J. 525: 25-30 (1999); arXiv:astro-ph/9906362;

[43] P.Szekeres, A Class of Inhomogeneus Cosmological Models, Commun.Math.Phys. 41, 55-64 (1974);

[44] D.A. Szafron, Inhomogeneous cosmologies: New exact solutions and their evolution, J. Math. Phys, 18, 1673 (1977);

[45] J.D.Barrow, J. Stein-Schables, Inhomogeneous cosmologies with cosmological constant, Phys. Lett. A, 103, (1984), 315;

[46] H. Stephani et al. Exact Solutions of Einstein's Field Equations, Second edition, Cambridge University Press (2003);

[47] S. W. Goode, J. Wainwright, Singularities and evolution of the Szekeres cosmological models, Phys.Rev. D26, 3315 (1982);

[48] A. Krasiński, Inhomogeneous cosmological models, Cambridge University Press (1997);

[49] J. Plebański, A. Krasiński, An introduction to General Relativity and Cosmology, Cambridge University Press (2006);

[50] K. Bolejko, A. Krasiński, C. Hellaby, M. Cèlèrier , Structure in the universe by exact methods Formation, Evolution, Interaction, Cambridge University Press (2010); 
[51] W.B.Bonnor, A.H.Sulainan, N. Tomimura, Szekeress space-times have no Killing vectors, Gen. Rel. Grav., 8, (1977), 549;

[52] N. Meures \& M. Bruni, Exact nonlinear inhomogeneities in $\Lambda$ CDM cosmology, Phys. Rev. D, (2011), 123519;

[53] M. Ishak, A. Peel, The growth of structures in the Szekeres inhomogeneous cosmological models and the matter dominated era, Phys.Rev. D85 (2012) 083502; arXiv:1104.2590 [astro-ph.CO];

[54] R. Sussman, Quasilocal variables in spherical symmetry: Numerical applications to dark matter and dark energy sources, Phys. Rev D 79, 025009 (2009);

[55] A. Peel, M. Ishak, M.A. Troxel, Large-Scale growth in the Szekeres inhomogeneous cosmological models with comparison to growth data, Phys. Rev. D86, (2012), 123508; arXiv:1212.2298 [astro-ph.CO];

[56] S. Weinberg, Gravitation and cosmology: principles and applications of the general theory of relativity, John Wiley \& Sons (1972);

[57] P.Sachs, Proc. R. Soc. London A, Gravitational Waves in General Relativity. VI. The Outgoing Radiation Condition, 264, (1961), 309;

[58] K. Bolejko, P. G. Ferreira, Ricci focusing, shearing, and the expansion rate in an almost homogeneuos Universe, JCAP 1205 (2012), 003; arXiv:1204.0909 [astro.ph-CO];

[59] T. Mattsson, Dark energy as a mirage, Gen. Rel. Grav. 42:567-599 (2010); arXiv:0711.4264 [astro-ph];

[60] M.Demianski et al., High-redshift cosmography: new results and implications for dark energy, MNRAS, 426, 1396 (2012);

[61] S. Capozziello et al. Comprehensive cosmographic analysis by Markov chain method, Phys. Rev. D 84, 124061, (2011);

[62] F.Y.Wang, Z.G.Dai, Shi Qi, Probing the cosmographic parameters to distinguish between dark energy and modified gravity models (Research Note), A\&A, 507, 53 (2009);

[63] V.Vitagliano et al. High-redshift cosmogaphy, JCAP03 (2010)005;

[64] S. Capozziello et al, Cosmography by gamma ray bursts. A\& A, 490, 31, (2008);

[65] D.Rapetti et al., A kinematical approach to dark energy studies, MNRAS, 375, 1510 (2008);

[66] D.J.Schwarz, B. Weinhost, (An)isotropy of the Hubble diagram: comparing hemispheres, A\&A, 474, 717 (2007); arXiv:0706.0165[astro-ph]. 